\title{
GASTO PRIVADO COM SAÚdE POR CLASSES DE RENDA
}

\author{
Samuel Kilsztajn*, Marcelo Bozzini da Camara, Manuela Santos Nunes do Carmo \\ Trabalho realizado no Laboratório de Economia Social do Programa de Estudos \\ Pós-Graduados em Economia Política - LES/PUCSP, S. Paulo-SP.
}

RESUMO - OBjetivo. 0 artigo analisa a participação do gasto privado com saúde das famílias no PIB e no total da renda familiar per capita e a distribuição do gasto privado com saúde das famílias por classes de renda.

Métodos. A pesquisa utilizou os microdados da Pesquisa Nacional por Amostra de Domićlios de 1998, com a divisäo da população em quatro classes de renda familiar per capita e a distribuiçäo dos gastos em planos de saúde, consultas médicas, consultas com outros profissionais de saúde, exames, medicamentos, artigos ortopédicos e aparelhos médicos, óculos e lentes, odontologia, hospitais, enfermagem domiciliar e outros gastos com saúde.

Resultados. Apenas $7,2 \%$ da população com renda familiar per capita até I salário mínimo em 1998 tinha direito a algum plano de saúde, e $\circ$ gasto privado com saúde das famílias desta classe, que representava $52,5 \%$ da população, era em média de $R \$ 5,36$ por pessoa. Para as pessoas com renda familiar per capita acima de 9 salários mínimos, os planos de saúde atingiam $83,2 \%$ da população e $\circ$ gasto privado com saúde das famílias $R \$ 133,04$.

Conclusöes. A implantação do Sistema Único de Saúde foi acompanhada pelo crescimento expressivo dos planos de saúde nos anos 90. Mesmo se todo 0 gasto público com saúde fosse destinado à população sem planos de saúde, ainda assim 0 gasto destas pessoas em 1998 só alcançaria $R \$ 352,62$, ou seja, $43 \%$ do gasto com saúde das pessoas com planos, $\mathrm{R} \$ 819,08$.

UNITERMOS: Economia da Saúde. Saúde pública. Gasto com saúde.

\section{INTRODUÇÃO}

Aimplantação do Sistema Único de Saúde (SUS) coincidiu com o corte das verbas públicas que acompanhou a política econômica antiestatizante e liberalizante do país nos anos 90. Auniversalização da assistência médica no Brasil, teoricamente, deveria elevar o grau de cobertura do sistema público de saúde. Concretamente, contudo, as restrições financeiras ea deterioração dos serviços públicos de saúde permitiram a segmentação do mercado e o crescimento expressivo da assistência médica suplementar no país ${ }^{1,2}$.

Ogasto privado com saúde é dividido entre gasto privado das famílias e gasto privado das empresas. Deacordo com os dados da Pesquisa Nacional por Amostra de Domicílios (PNAD) ${ }^{3}$, podemos estimar em $\mathrm{R} \$ 37,9$ bilhões o gasto privado anual com saúde das famílias em 1998, que incluigastos com planos de saúde, consultas médicas, consultas com outros profissionais de saúde, exames, medicamentos, artigos ortopédicos e aparelhos médicos, óculos e lentes, odontologia, hospitais, enfermagem domiciliar e outros gastos com saúde.

*Correspondência:

R. Marquês de Paranaguá, 164/602

01303-050 - São Paulo - SP - Tel.:(1 1) 3256-6996 skil@pucsp.br
O Produto Interno Bruto (PIB) brasileiro em 1998 foi estimado em $R \$ 899,8$ bilhões a preços correntes (Bacen, 2000: 15) ${ }^{4}$. A participação do gasto privado com saúde das famílias, desta forma, pode ser estimada em $4,2 \%$ do PIB de 1998.

Corrêa et al. ${ }^{5}$ estimaram em $3,6 \%$ o gasto privado com saúde das famílias em relação ao PIB de 1996. Para esta estimativa, os autores utilizaram ogasto direto das famílias com saúde da Pesquisa de Orçamento Familiar (POF) do IBGE nas nove regiốes metropolitanas, Brasília e Goiânia (extrapolada para o Brasil).

Ogasto privado comsaúdedasempresas, por suavez, foi stimado em $0,7 \%$ do PIB de $1996^{5}$. A partir das estimativas do gasto privado com saúde das famílias em 1998 da PNAD/98 e do gasto privadocomsaúdedasempresas para 1996, podemos estimar o gasto privado total com saúde de 1998 em R\$44,2 bilhões ou 4,9\% do PIB-oBanco Mundial (2000) ${ }^{6}$ estimouem 4\%aparticipação do gasto privado comsaúdeno PIB do Brasilem 1998.

A partir da PNAD do Instituto Brasileiro de Geografia e Estatística (IBGE) ${ }^{3}$, o presente artigo analisa a participação do gasto privado com saúde das famílias no PIB e na renda familiar per capita por classes de renda; e a distribuição do gasto privado com saúde das famílias entre categorias de gasto com saúde.

No decorrer deste trabalho, faremos ain- da algumas considerações sobre a distribuição entre gasto público e privado com saúde no Brasil e em alguns países selecionados.

\section{Métodos}

APNAD levantou em 1998 as características de saúde dos moradores que incluem informações sobre o grau de cobertura dos planos de saúde e o gasto privado com saúde das famílias distribuído em I I categorias: planos de saúde, consultas médicas, consultas com outros profissionais de saúde, exames, medicamentos, artigos ortopédicos eaparelhos médicos, óculos e lentes, odontologia, hospitais, enfermagem domiciliar e outros gastos com saúde.

Aamostra da PNAD de 1998 foi composta por 344.975 pessoas que, a partir do processo de expansão da amostra, correspondem a 158,2 milhões de brasileiros (excluída a população rural da antiga Região Norte).

A partir dos microdados da PNAD, o gasto privado total com saúde das famílias foi anualizado, considerando-se que o gasto em medicamentos refere-se a gastos no mês de setembro de 1998 (30 dias) e o gasto nas demais categorias refere-se a gastos entre juIho e setembro de 1998 (três meses). Dado que a maior parte do gasto privado com saúde das familias foi realizado entre julho e setembro de 1998, e que o deflator implícito do PIB 
foi de $4,3 \%$ durante todo o exercício de $1998^{4}$, não se fazem necessários ajustes para o cálculo da participação percentual do gasto privado com saúde das famílias no PIB.

A distribuição pessoal da renda pode ser apresentada, de forma geral, como: a) distribuição do rendimento entre pessoas economicamente ativas; b) distribuição do rendimento entre famílias; ec) distribuição do rendimento familiar per capita da população. Para a análise do nível de bem-estar da população, a distribuição do rendimento familiar per capita da população é a mais apropriada ${ }^{7}$.

APNAD permite dividir a população brasileira por classes de renda familiar per capita. Neste trabalho utilizamos quatro classes de renda familiar mensal per capita: até I salário mínimo; mais de I a 3 salários mínimos; mais de 3 a 9 salários mínimos; e mais de 9 salários mínimos. O presente artigo analisa a participação do gasto privado com saúde das famílias no total da renda e a distribuição do gasto privado com saúde das famílias entre as I I categorias pesquisadas pela PNAD nas quatro classes de renda familiar per capita acima definidas.

\section{Resultados E DISCUSSÃO}

\section{Distribuição da renda familiar per capita}

Dos I58,2 milhões de brasileiros em 1998 (excluída a população rural da antiga Região Norte), nossa distribuição da renda familiar per capita inclui I 53, I milhões de pessoas. As 5,2 milhões de pessoas excluídas da nossa análise correspondem a pessoas em famílias sem declaração de renda familiar (4 milhões), agregados ( 0,6 milhões), pensionistas $(0, I \mathrm{mi}-$ Ihões) e empregados domésticos e parentes de empregados domésticos ( 0,4 milhões).

A renda familiar per capita destes 153,1 milhões de pessoas atingia uma média mensal de $R \$ 253,95$ em 1998, mas a mediana registrava apenas $R$ \$ 126,67 . Para a distribuição da renda familiar mensal per capita, estes I53, I milhões de pessoas foram divididos nas quatro classes mencionadas: até I salário mínimo (classe I); mais de I a 3 salários mínimos (classe 2); mais de 3 a 9 salários mínimos (classe 3); e mais de 9 salários mínimos (classe 4).

Deacordo coma Tabela I, mais dametadeda população $(52,5 \%)$ recebiaaté $R \$ 130,00$ derenda familiar per capita, ou seja, um salário mínimo em 1998. A classe 2, que recebia mais de $\mathrm{R} \$ 130,00$ a
$\mathrm{R} \$ 390,00$ por pessoa, somava $31,7 \%$ da população; a classe 3, que recebia mais de $R \$ 390,00 \mathrm{a} R \$$ I. I70,00 percapita, somava I2,7\% da população; e a classe 4 , que recebia mais de $R \$ 1.170,00$ per capita, somava 3,2\% da população.

Éimportante considerarqueapenas $3,2 \%$ da população brasileira, que correspondem a 4,8 milhões de pessoas, pertenciam a famílias com uma renda familiar per capita superior a $\mathrm{R} \$$ I. 170,00.Umafamília compostapor três pessoas com uma renda familiar superior a $\mathrm{R} \$ 3.50 \mathrm{I}, 00$ em 1998, estaria incluída na classe 4 (o tamanho médio dafamíliana classe 4 erade 2,5 pessoas em 1998). Cabe lembrar, como salienta Hoffmann ${ }^{7}$, que pessoas relativamente ricas para a distribuição de renda no Brasil costumam se considerar "pobres" ou, quando muito, "classe média".

Aclasse 4 representava 3,2\% da população, mas recebia $27,0 \%$ do total da renda de todas as classes, enquanto a classe I, que representava $52,5 \%$ da população, recebia apenas $13,2 \%$ do total da renda de todas as classes.

\section{Renda familiar e distribuição do gasto privado com saúde das famílias por categorias}

Em 1998, o gasto privado anual com saúde das famílias foi de $R \$ 37,9$ bilhões para o total de 158,2 milhões de pessoas e de $\mathrm{R} \$ 35,8$ bilhões para os I53, I milhões de pessoas com renda familiar per capita declarada.

Estes $\mathrm{R} \$ 35,8$ bilhões correspondem a um gasto privado mensal médio com saúde per capita de R\$ 19,49 para o conjunto da população. A classe I registrou um gasto privado mensal médio per capita com saúde de $R \$$ 5,36 ; a classe 2 de $R \$ 18,92$; a classe 3 de $R \$$ 5I,27; e a classe 4 de $R \$ 133,04$ (Tabela I).

Embora com valores absolutos tão díspares, a participação percentual do gasto privado com saúde das famílias no total da renda familiar é relativamente estável $(8,4 \%$ a $8,1 \%$ da renda familiar) para as três primeiras classes de renda que representavam $96,8 \% \mathrm{da}$ população. $O$ percentual de gasto privado com saúde das famílias na renda só é relativamente menor para a classe 4 , com 6, $1 \%$.

Vejamos agora como é distribuído o gasto privado com saúde das famílias por categorias de gasto com saúde nas 4 classes de renda familiar per capita.

Dos $R \$ 5,36$ per capita que a classe I gastou por mês em 1998, os medicamentos somava $R \$ 3,46$, ou seja, $64,6 \%$ do total. Para a classe I, ogastocomplanosdesaúdesomava $8,9 \%$ dogasto total com saúde, tratamento dentário e próteses $6,6 \%$ e os demais gastos somavam os restantes 19,9\% do gasto total com saúde (Gráfico I).

À medida que sobe a renda familiar per capita, cai a participação do gasto com medicamentosesobea participaçãodogasto complanos de saúde e com odontologia no gasto total com saúde das famílias. Mas, sea participação do gasto com medicamento no gasto total com saúde cai com o nível de renda, isto não significa dizer que o gasto absoluto commedicamentosacompanha esta queda. As famílias da classe I gastavam $R \$$ 3,46 per capita com medicamentos ao mês enquanto as famílias da classe 4 gastavam $R \$ 27,36$. Com mensalidades de planos de saúde per capita as famílias da classe I gastavam $R \$ 0,48$ e as famílias da classe 4 R\$ 6I,73 (Tabela I).

É importante esclarecer que o gasto com medicamentos per capita, assim como os demais gastos com saúde, foi cal culado a partir do total do gasto com medicamentos e do número de pessoas em cada uma das classes, independentemente do número de pessoas que efetivamente realizou o gasto. Desta forma, $R \$ 3,46$ correspondiam ao gasto médio com medicamentos assim como $\mathrm{R} \$ 0,48$ correspondia ao gasto médio com planos de saúde de cada uma das pessoas na classe I, dado que apenas $7,2 \%$ das pessoas desta classe possuíam planos de saúde em 1998 (Tabela I). O percentual de pessoas que possuía planos de saúde em 1998 sobe para $31,2 \%$ para a classe $2,63,1 \%$ para a classe 3 e $83,2 \%$ para a classe 4 . As famílias das classes 3 e 4 gastavam com mensalidade de planos mais de $40 \%$ do gasto total com saúde. Para estas duas classes, o gasto com medicamentos constituía a segunda maior categoria de gasto privado com saúde das famílias $(29,9 \%$ para a classe 3 e $20,6 \%$ para a classe 4 ) e o gasto com tratamento dentário e próteses a terceira maior categoria de gasto com saúde das famílias (I2,9\% e I5,3\%, respectivamente) (Gráfico I).

\section{Distribuição entre gasto público e privado com saúde}

O gasto privado com saúde foi estimado em R\$ 44,2 bilhões ou 4,9\% do PIB de 1998. As estimativas para o gasto público com saúde em 1998 do Ministério da Saúde $(2000)^{8}$ são de $R \$ 29,7$ bilhões correntes, distribuídos entre gasto federal, estadual e municipal que, considerando-se a estimativa do PIB de R\$ 899.814 milhões para 1998 (BACEN, 2000: I5), corresponde a 3,3\% do PIB (Tabela 2). A 


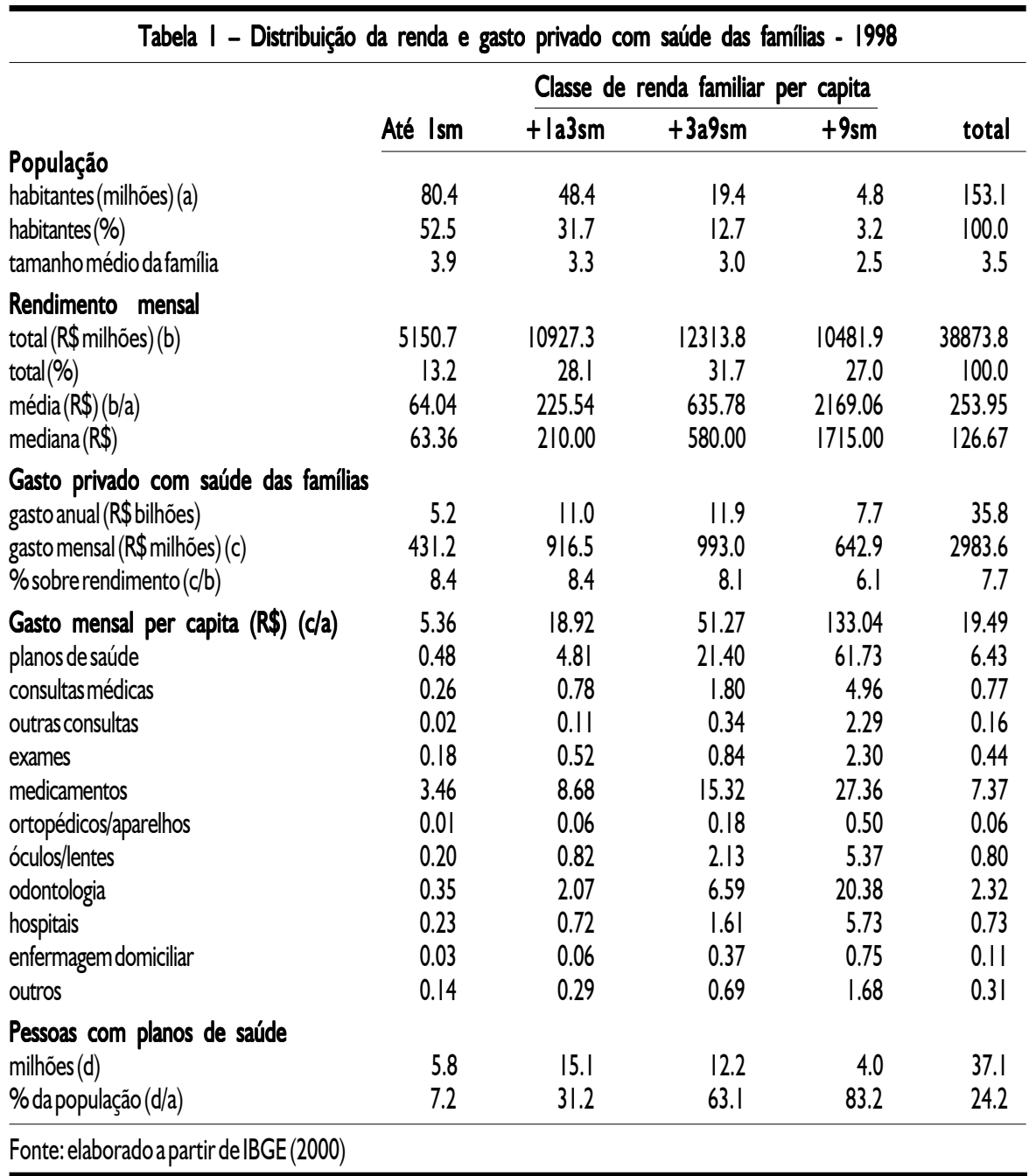

Gráfico I - Distribuição do gasto privado com saúde das famílias por categoria-Brasil 1998

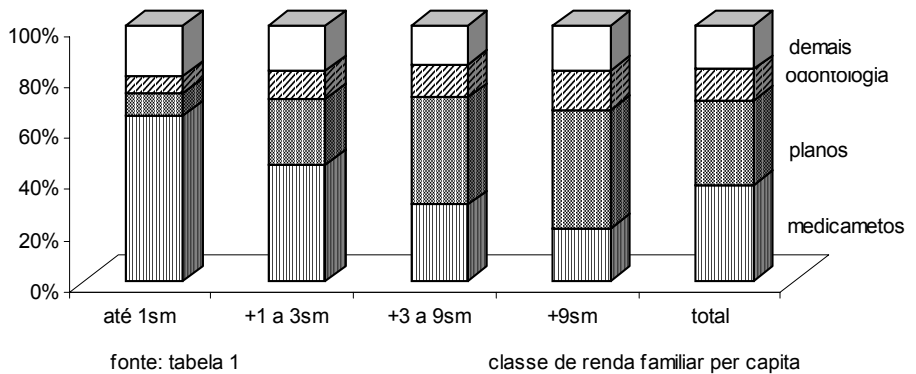

estimativa do Ministério da Saúde $(2000)^{8}$ é consistente com o trabalho de Fernandes? que estimou o gasto público com saúde em $3,4 \%$ do PIB de 1995 .

As estimativas para gasto público e privado com saúde no Brasil totalizam um gasto total de
Como não é possível distribuir o gasto privado com saúde das empresas e o gasto público com saúde por classe de renda familiar per capita, utilizaremos um corte alternativo para a continuidade de nossa análise.

SabemosqueaimplantaçãodoSistemaÚnico de Saúde foi acompanhada pelo crescimento expressivo dos planos de saúde, e que o grau de coberturados planos desaúdecrescecomarenda da população (de 7,2\% na classe I para 83,2\% na classe4). Destaforma, alternativamenteàdistribuição por classes de renda familiar per capita, dividimos a população brasileira em dois grupos, 0 primeirosemdireitoaplanosdesaúdeeosegundo com direitoaalgum planodesaúde. Nosso universo, comesteexpediente, passaa incluir as pessoas emfamíliassem declaraçãoderendafamiliar, agregados, pensionistas, empregados domésticos e parentes de empregados domésticos, I58,2 miIhões de brasileiros (excluída a população rural da antiga Região Norte), com um gasto total privado com saúde das famílias de 37,9 bilhões.

Os planos de saúde são a segunda maior categoria de gasto privado com saúde das famílias, I,4\% do PIB (Tabela 2). A primeira categoria é constituída pelo gasto com medicamentos, I,6\% do PIB. Vale lembrar que embora $\circ$ gasto com medicamento per capita tenha registrado $R \$ 61,00$ para as pessoas sem planos de saúde e $R \$ 175,99$ para as pessoas com planos, $51,7 \%$ do gasto total com medicamentos é de responsabilidade da população não coberta por planos de saúde.

Considerando-se que os gastos privados com saúde das empresas respondem por $0,7 \%$ do $\mathrm{PIB}$, o gasto privado total com planos de saúde pode ser estimado em 2, $1 \%$ do PIB em 1998. Os planos de saúde, jurídicoinstitucionalmente, podem ser divididos em medicina de grupo, autogestão, cooperativas e seguradoras que, em 1999, detinham respectivamente, $39 \%, 25 \%, 24 \%$ e I3\% da assistência médica suplementar do país ${ }^{10}$.

Assumimos, para efeito de análise, que o gasto privado com saúde das empresas dirigese essencialmente para as pessoas com planos de saúde e o gasto público com saúde dirige-se essencialmente para as pessoas sem planos de saúde. Este procedimento ainda superestima o gasto público com saúde per capita para as pessoas sem planos de saúde, na medida em que parte do gasto público com saúde atinge também as pessoas com planos de saúde.

De acordo com a Tabela 2, o gasto total 


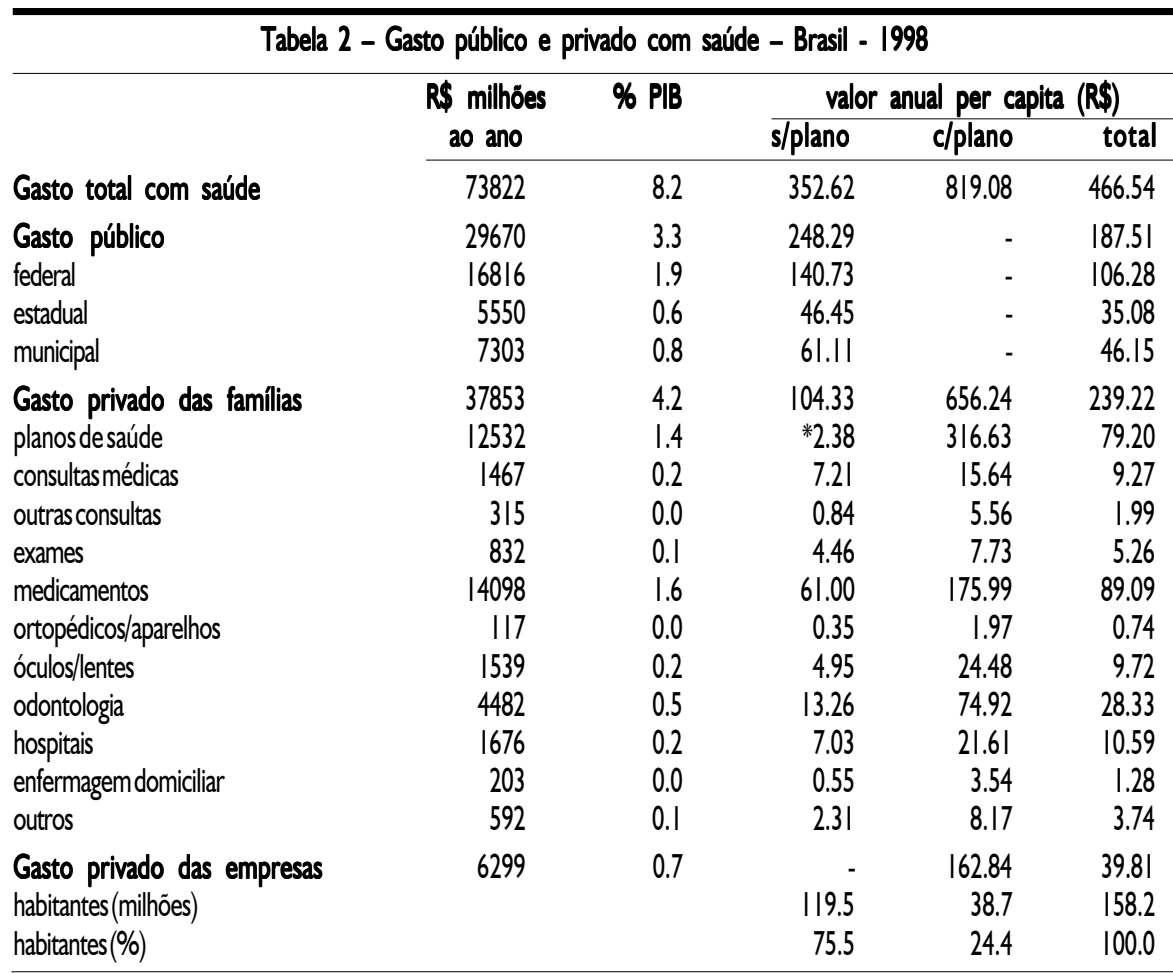

Fonte: elaboradoa partir de Ministério da Saúde(2000), IBGE(2000)e Corrêa/Piola/Arilha(1998). * Emalgumasfamílias, a pessoa de referência, mesmo sem plano de saúde, paga as despesas com saúde de algum membro dafamília com plano de saúde.

Tabela 3 - Análise comparativa de gasto público e privado com saúde - 1997

\begin{tabular}{|c|c|c|c|c|c|c|c|c|c|}
\hline & \multicolumn{3}{|c|}{ PPC per capita/ano } & \multicolumn{3}{|c|}{$\%$ do PIB } & \multicolumn{3}{|c|}{ distribuição (\%) } \\
\hline & público & privado & total & público & privado & total & público & privado & total \\
\hline Argentina & 503 & 663 & 1166 & 4.2 & 5.6 & 9.8 & 43.2 & 56.8 & 100.0 \\
\hline Brasil & 233 & 270 & 503 & 3.4 & 3.9 & 7.3 & 46.3 & 53.7 & 100.0 \\
\hline USA & 1913 & 2208 & $4|2|$ & 6.5 & 7.5 & 13.9 & 46.4 & 53.6 & 100.0 \\
\hline México & 221 & 148 & 369 & 2.8 & 1.9 & 4.7 & 60.0 & 40.0 & 100.0 \\
\hline Canadá & 1505 & 653 & 2158 & 6.4 & 2.8 & 9.2 & 69.8 & 30.3 & 100.0 \\
\hline França & 1504 & 522 & 2026 & 7.1 & 2.5 & 9.6 & 74.2 & 25.8 & 100.0 \\
\hline Espanha & 900 & 282 & 1182 & 5.6 & 1.8 & 7.4 & 76.1 & 23.9 & 100.0 \\
\hline Alemanha & 1822 & 542 & 2364 & 8.3 & 2.5 & 10.7 & 77.1 & 22.9 & 100.0 \\
\hline ReinoUnido & 1177 & 214 & |39| & 5.8 & 1.0 & 6.8 & 84.6 & 15.4 & 100.0 \\
\hline
\end{tabular}

anual com saúde per capita em 1998 foi de $R \$$ 466,54 (R\$ 187,5I público, R\$239,22 das famílias e $R \$ 39,81$ das empresas). Mesmo que todo o gasto público com saúde tivesse sido destinado à população sem planos de saúde, 0 gasto total anual com saúde per capita destas pessoas teriasido $R \$ 352,62$ ( $R$ \$248,29 público e $R \$ 104,33$ privado) enquanto que, para a população com planos de saúde, só o gasto privado anual com saúde atingiu $R \$ 819,08(R \$$ 656,24 das famílias e $R \$ 162,84$ das empresas).
Apresentamosna Tabela 3 as estimativas do Banco Mundial ${ }^{6}$ para gastos público e privado em valor e em percentual do PIB; e a distribuição do gasto público e privado no gasto total com saúde para 1997 de alguns países latinoamericanos (Argentina, Brasil e México) eindustrializados (Alemanha, Canadá, Espanha, França, Estados Unidos e Reino Unido). Para comparações internacionais de bem-estar deve-se utilizar a PPC (paridade do poder de compra)e não o dólar americano, dado que o poder de compra do dólar americano varia de acordo com a taxa de câmbio de cada país (Summers) Heston, (99I)". Os países na Tabela 3 foram ordenados de acordo com a distribuição do gasto com saúde em público e privado.

Nos países latino-americanos e nos Estados Unidos, o gasto público registrava $43 \%$ a $60 \%$ do gasto total com saúde, enquanto que para os demais países industrializados esta participação atingia $70 \%$ a $85 \%$.

Os Estados Unidos, particularmente, embora apresentem uma baixa participação do gasto público no gasto total com saúde, atingem elevada participação do gasto público em relação ao $\mathrm{PIB}(6,5 \%)$, superior mesmo à do Canadá, Espanha e Reino Unido. Nos países latinoamericanos a participação do gasto público em relação ao PIB registrava 2,8\% a 4,2\% em 1997.

Vale a pena deter-se um pouco nos Estados Unidos, conhecido pelo seu modelo de assistência privada à saúde. Os planos privados de saúde cobrem quase $75 \%$ da população norte-americana, mas existe sobreposição entre cobertura pública e privada. A partir de dois programas básicos, Medicare e Medicaid, que somavam $74 \%$ de todo o gasto público com saúde em 1998, o setor público atende a população acima de 65 anos, deficientes, doentes renais crônicos e pessoas de baixa ren$\mathrm{da}^{12}$. O Medicaree Medicaid cobrem, respectivamente, $14 \%$ e $13 \%$ da população, mas existe também sobreposição entre os dois programas. Por outro lado, 15\% da população não contam com cobertura privada nem são elegíveis para os programas públicos de saú$\mathrm{de}^{2}$. Entretanto, dada as características das pessoas atendidas pelo setor público (idosos, deficientes e pessoas de baixa renda), o gasto público representava, em $1998,45 \%$ do gasto total com saúde, $53 \%$ do gasto com atendimento domiciliar (home health care), $60 \%$ do gasto com enfermagem domiciliar e $61 \%$ do gasto com atendimento hospitalar (Tabela 4).

É importante esclarecer que nas Tabelas I e 2 considera-se como gasto com planos privados de saúde das famílias as mensalidades dos planos. Na Tabela 4, alternativamente, as mensalidades com os planos privados de saúde estão distribuídas entre os dispêndios efetuados pelos planos (atendimento médico, hospitais etc.) e o custo líquido dos planos privados (isto é, mensalidades menos dispêndios) registrados na rubrica administração. 


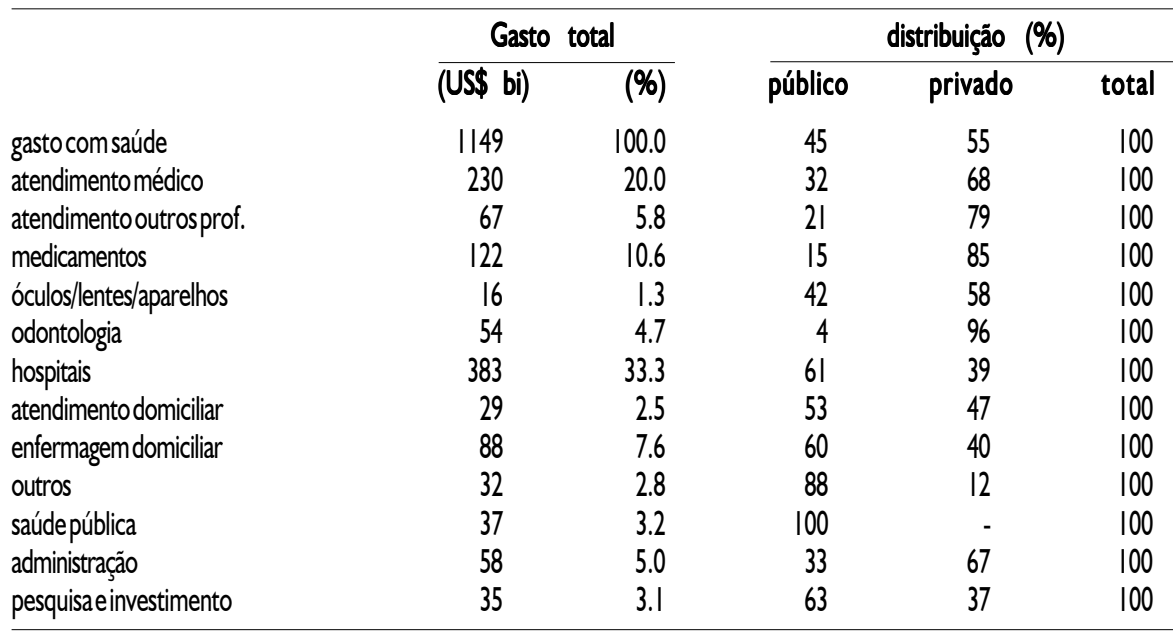

Fonte: elaboradoa partirdeUS-HCFA(200I).
Tabela 4 - Gasto público e privado com saúde - Estados Unidos - 1998

1988 denominated "Sistema Unico de Saude" was followed by an expressive expansion of private insurance coverage in the 1990's. Even if all public health expenditures had been exclusively directed to the population without any private insurance, these people's health expenditures would only reach $43 \%$ of the health expenditures of those with private insurance. [Rev Assoc Med Bras 2002; 48(3): 258-62]

KeY Words: Health economics. Health expenditures. Private health expenditures.

\section{RefERÊNCIAS}

I. Baer W, Campino AC, Cavalcanti T. Condições e política de saúde no Brasil: uma avaliação das últimas décadas. Econom Aplicada (São Paulo)2000; 4:763-85.

2. Almeida C.O mercado privado de serviços de saúde no Brasil: panorama atual e tendências da assistência médica suplementar. Brasília: IPEA; 1998. (Texto para Discussão n.599). Disponível em: URL: http://www.ipea.gov.br.

\section{Conclusões}

Aimplantação do Sistema Único de Saúde foi acompanhada pelo crescimento expressivo dos planos de saúde nos anos 90 .

Apenas $7,2 \%$ da população com renda familiar per capita até I salário mínimo em 1998 tinha direito a algum plano de saúde e o gasto privado com saúde das famílias desta classe, que representava $52,5 \%$ da população, era em média de $R \$ 5,36$ por pessoa. Com a elevação do nível de renda, sobe tanto o grau de cobertura dos planos de saúde como o gasto privado com saúde das famílias. Para as pessoas com renda familiar per capita acima de 9 salários mínimos, os planos de saúde atingiam $83,2 \%$ da população e o gasto privado com saúde das famílias $R \$ 133,04$.

Mesmo se todo o gasto público com saúde fosse destinado à população sem planos de saúde, ainda assim o gasto destas pessoas em 1998 só alcançaria $R \$ 352,62$, ou seja, $43 \%$ do gasto com saúde das pessoas com planos, $R \$$ 819,08. De acordo com Porto ${ }^{13}$, para garantir a eqüidade, os recursos para a saúde deveriam ser distribuídos através de uma discriminação positiva em favor dos mais desfavorecidos. Neste sentido, para promover a justiça social, é necessário mensurar e atender a demanda por serviços de saúde da população pobre do país que, dado o perfil concentrador de renda do modelo econômico brasileiro, énecessariamente superior à demanda por serviços de saúde das camadas privilegiadas pelo sistema.

\section{Agradecimentos}

Agradecemos a colaboração de Anacláudia Rossbach e Rodolfo Hoffmann.

\section{SUMMARY}

Private health eXpenditures and INCOME DISTRIBUTION IN BRAZIL

BACKGROUND. Thispaperanalysestheshare of the family private health expenditures in the Brazilian GDP and in personal income; and the distribution of the family private health expenditures amongsocialgroups.

Methods. The research utilized the 1998 Brazilian HomeSampleSurvey (Pesquisa Nacional por Amostra de Domicilios) with the division of the population into four social groups according to the family income per capita; and the distribution of the family private health expenditures among health insurance, physicians, other health professionals, medical tests, drugs, orthopedic and other medical durables, vision products, dental services, hospital care, nursing home care and other health spending.

Results. In 1998, only $7.2 \%$ of the population with familyincome percapitaup to I minimum wage had healthinsuranceand the healthexpenditures of this group, that represented $52.5 \%$ of the population, was US\$4.62 percapita. Forthepeople with 9 and more minimum wages per capita the health insurance coverage was $83.2 \%$ and the healthexpenditureswas US\$1/4.66 per capita.

CONCLUSIONS. The implementation of the Brazilian public universal health care system in
3. IBGE. Pesquisa nacional por amostra de domicílios-microdados. Rio de Janeiro: 2000 IBGE.(CD-ROM).

4. Bacen (Banco Central do Brasil).Relatório anual I 999. Brasília: BACEN; 2000.

5. Corrêa S, Piola S, Arilha M.Cairo em ação: estudo de caso Brasil. New York: Population Reference Bureau; 1998. (mimeo)

6. Banco Mundial. World development indicators. Washington: World Bank; 2000.(CD-ROM).

7. Hoffmann R. Distribuição da renda no Brasil: poucos com muito e muitos com muito pouco. In: Dowbor L, Kilsztajn S, organizadores. Economia social no Brasil. São Paulo:SENAC; 2001 .

8. Ministério Da Saúde. Estimativas de impacto da EC n.29/00. Brasília: SIOPS; 2000.

9. Fernandes MAC, Rocha DCC, Oliveira MMS, RibeiroJAC, Aquino LMC. Gasto social das três esferas de governo - 1995. Brasília: IPEA(Texto para Discussão n.598). Disponível em: URL: http://www.ipea.gov.br. Acessoem: julho 2002.

10. Ciefas (Comitê de Integração de Entidades Fechadas de Assistência à Saúde). A história da autogestão em saúde no Brasil. São Paulo: CIEFAS; 2000.

II. Summers R, Heston A. The penn world table (mark5): an expanded set of international comparisons 1950-1988. QJ Econ 1991; 106:327-68.

12. US - HCFA (Health Care Financing Administration). National Health Expenditures Tables (tables 3 and I0). Disponível em: URL:http:// www. hcfa.gov/stats/nheoact/tables/. Acesso em: março $200 \mathrm{I}$

13. Porto SM. Justiça social, equidade e necessidade em saúde. In: Piola SF, Vianna SM, organizadores. Economia da saúde: conceito e contribuição para a gestão da saúde. Brasília: IPEA; 1998. p. 123-40.

Artigo recebido:30/10/200

Aceito para publicação: 13/02/2002

Rev Assoc Med Bras 2002; 48(3): 258-62 